\title{
Family and Peer Relationships in a Residential Youth Sample: Exploring Unique, Non-Linear, and Interactive Associations with Depressive Symptoms and Suicide Risk
}

\author{
Alannah Shelby Rivers $\mathbb{B}^{1} \cdot$ Jody Russon ${ }^{2} \cdot$ Payne Winston-Lindeboom ${ }^{1} \cdot$ Linda Ruan-lu $^{1} \cdot$ Guy Diamond ${ }^{1}$
}

Received: 20 August 2021 / Accepted: 5 October 2021 / Published online: 13 October 2021

(c) The Author(s), under exclusive licence to Springer Science+Business Media, LLC, part of Springer Nature 2021

\begin{abstract}
Close relationships are consequential for youth depressive symptoms and suicide risk, but nuanced research examining intersecting factors is needed to improve identification and intervention. This study examines a clinical, residential sample of 939 adolescents and young adults ages 10 to 23 years old $(M=15.84, S D=1.53 ; 97.7 \%$ white, $99.5 \%$ non-Hispanic, $55 \%$ female). The final model found that family conflict, parental criticism, verbal bullying, and interactions with friends were associated with depressive symptoms in the expected directions, and there were significant interactions with family, peer, and demographic variables. However, most associations with suicide risk were indirect. Associations involving family factors, peer factors, depressive symptoms, and suicide are not always straightforward, and should be understood within a microsystemic context.
\end{abstract}

Keywords Bullying $\cdot$ Depression $\cdot$ Family environment $\cdot$ Friendships $\cdot$ Suicide

\section{Introduction}

Suicide is the second leading cause of death among ages 10 to 34 and a major crisis among adolescents and young adults (National Center for Injury Prevention and Control (U.S.). Division of Violence Prevention, 2015). Although the causes for suicide are multifactorial, most cases are linked to psychopathology (Gould \& Kramer, 2001), and particularly to depression. As depression also continues to rise among adolescents and young adults (Mojtabai et al., 2016), it is important to develop an understanding of factors that may contribute to, or buffer against, depressive symptoms and/or suicide risk in order to prevent the continued acceleration of these interconnected threats. Social relationships with family and peers have been identified as particularly important categories of risk and protective factors (e.g., Sun \& Hui,

Alannah Shelby Rivers

asr357@drexel.edu

1 Center for Family Intervention Science, Drexel University, 3141 Chestnut St, Philadelphia, PA 19104, USA

2 Department of Human Development and Family Science, Virginia Polytechnic Institute and State University, Blacksburg, VA, USA
2007), but most research has examined these factors as a few independent indicators of risk, rather than as a complex and interactive microsystem. This method limits both theoretical understanding and applicability of findings to improvements in identification and treatment of at-risk youth. Therefore, the current study seeks to explore unique and complex associations (such as non-linear associations or interactions) between family and peer factors with depressive symptoms and suicide risk in a high-risk residential sample.

The microsystemic social environments of adolescents and young adults have a profound effect on psychological development (Vieno et al., 2007). Ecological models (e.g., Bronfenbrenner, 1977) of development and health illustrate the influence of context on individual health and psychosocial well-being. Such models encourage the examination of both proximal and distal factors surrounding a person to understand the interrelatedness of multiple embedded systems of influence (e.g., culture, society, neighborhood, family networks). Extant research suggests that there are two critical microsystems that are especially important for understanding common but pervasive mental health symptoms (e.g., depressive symptoms, suicide risk) in adolescents: the family and the peer group. The quality of relationships with family and peers are particularly potent factors contributing to risk for depression and suicide (Diamond et al., 2021). Close and 
trusting relationships with family members and peers build support (Bögels \& Brechman-Toussaint, 2006; Drake \& Ginsburg, 2012), facilitate coping (Compas et al., 2017), and promote a sense of belonging (Vitaro et al., 2009). The opposite is also true: when adolescents and young adults experience conflict with their families and isolation from peers, this contributes to stress and impacts psychosocial functioning (Orben et al., 2020; Sheeber et al., 2001). Indeed, family and peer factors have been associated not only with the development of clinically-relevant symptoms (Prinstein et al., 2000) but also with treatment trajectories and outcomes (Baker \& Hudson, 2013; Rapp et al., 2021).

Family factor research has identified several specific and potentially important risk factors that may help prevent or contribute to the development of depressive symptoms and associated suicide risk in adolescents and young adults. First, parental criticism can be a potent risk factor; youth who perceive high levels of parental criticism are at increased risk for depression (Rapp et al., 2021) and suicidal thoughts and behaviors (Campos et al., 2013). Similarly, conflict in the home is robustly associated with depressive symptoms (Rice et al., 2006) and suicide risk (Randell et al., 2006). Furthermore, a lack of perceived parental support is consistently associated with risk for developing depression (Baetens et al., 2015) as well as risk for suicide attempts (Sheftall et al., 2013). Finally, youth who perceive less parental monitoring (e.g., not being aware of the youth's whereabouts) may be at greater risk for depression (Yu et al., 2006). Importantly, longitudinal studies suggest that psychological symptoms often follow, not precede, these types of family factors (Cummings et al., 2015). That is, family conflict and criticism may be risk factors (and family support and monitoring protective factors) for later development of psychological symptoms, and these associations are not merely reflecting deterioration in close family relationships following the onset of symptoms.

Positive and negative experiences with peers can also influence psychological well-being, including risk for depressive symptoms and suicide. Positive experiences, such as friendships, have small, but consistent, negative associations with depressive symptoms (Schwartz-Mette et al., 2020). Longitudinal research suggests that high-quality friendships may protect against later depression symptoms (Jacobson \& Newman, 2016), whereas lack of friendships and feelings of isolation may damage youth psychosocial health (Ueno, 2005; Vitaro et al., 2009). Negative experiences, such as bullying (verbal, physical, and cyberbullying) are also associated with depressive symptoms and suicide risk over time (Brunstein Klomek et al., 2007; KaltialaHeino et al., 2009). Notably, it is important to distinguish between different types of bullying, such as physical and cyberbullying, which may have different associations with depressive symptoms (Wang et al., 2011).
However, these family and peer factors should not be understood as simply a collection of factors that may be added and subtracted to understand individual risk of depressive symptoms or suicide. First, much of this research has examined family and peer factors separately, without accounting for possible overlap (unique associations). This makes it difficult to determine which factors may be the most important. Second, and even more crucially, more complex effects like interactions and non-linear associations have been underexplored. While many studies suggest direct, linear relationships between family and peer factors and youth mental health, others suggest that, in actuality, these mechanisms interact in complex ways (Bradley \& Corwyn, 2000; Ciairano et al., 2007). For example, one study found that supportive peer relationships were associated with lower depressive symptoms only under conditions of low family support. These supportive peer relationships were associated with an increase in depressive symptoms for adolescents with a high degree of family support (Barrera \& Garrison-Jones, 1992). Interestingly, other studies have found the opposite: supportive peer relationships were only associated with lower depressive symptoms under conditions of low family conflict (Ciairano et al., 2007). Some studies also suggest that family factors may have curvilinear, rather than linear, associations with psychological outcomes. For example, poor family control (e.g., lack of parental monitoring) may be a risk factor for poor adjustment, but higher levels of family control have diminishing returns (Kurdek \& Fine, 1994). Therefore, examining factors in isolation and excluding the possibility of non-linear and interactive associations may lead to incorrect conclusions about the role of family and peer factors in depressive symptoms and suicide risk.

There is a clear need for a detailed examination of potentially unique, non-linear, and interactive associations between family and peer factors with depressive symptoms and suicide risk. However, for this examination to be meaningful for research, theory, and practice, it is important to account for a few additional considerations. First, studies suggest the effects of family and peer factors on youth mental health may be moderated not only by other social relationship factors but also by demographic factors. Gender appears to be a particularly salient moderator in previous research (Kerr et al., 2006; Lewis et al., 2015); depressive symptoms are more common among girls than boys, and differentiated social roles for boys and girls may result in family and peer factors affecting youth differently (Parker \& Brotchie, 2010). Second, the majority of the research that examines combined and complex effects of family and peer factors has only considered depressive symptoms, not suicide risk (Hawton et al., 2013). As these outcomes are often linked, it is critical to determine whether these family and peer factors are uniquely related to suicide or primarily through increases in depressive symptoms. Finally, these associations are particularly important to explore in 
higher-risk clinical populations, due both to the severity of risk and the potential for differences in how family and peer factors are associated with risk in clinical samples, compared to more general youth and young adult samples (e.g., Queen et al., 2013).

\section{Current Study}

Taken together, it is important to examine previously-identified family and peer factors (parental monitoring, family support, family conflict, parental criticism, frequency of interactions with friends, verbal bullying, physical bullying, and cyberbullying), with the expectation that they will each be associated with depressive symptoms and/or suicide risk in adolescents and young adults. Furthermore, based on previous research and theoretical understanding, non-linear and interactive effects are anticipated between these factors, which must be understood to draw conclusions about the true effects of these family and peer factors. Based on previous research, interactions are also anticipated with demographic factors, which may shed light on which family and peer factors may be uniquely important for certain demographic groups. Finally, given the association between depressive symptoms and suicide risk, at least some associations between family and peer factors with suicide risk are expected to be mediated by depressive symptoms.

\section{Method}

\section{Participants}

The final sample consisted of 939 adolescents and young adults ages 10 to 23 years old $(M=15.84, S D=1.53)$. Among the 1,550 residential patients who opened the survey, 31 patients were removed from analysis because they completed no survey items, and 567 were removed from analysis because they had missing gender information due to a survey administration error. Participants missing the gender question did not significantly differ on depressive symptoms or suicide risk. The sample was approximately $97.7 \%$ white, $99.5 \%$ nonHispanic, 55\% female and $45 \%$ male ( $0.1 \%$ non-binary).

\section{Procedure}

The de-identified data used in this current study comes from a larger, quality improvement project at a privately-owned multisite psychiatric residential treatment center, which provides both outpatient and inpatient care to youth with different and co-occurring conditions (e.g., depression, anxiety, and substance use). Data were collected from 2019 to 2020. Staff administered the assessment battery at the intake meeting using the electronic BH-Works platform (www.mdlogix.com).
The assessment takes approximately 15 minutes, and scores are automatically computed and uploaded into patients' electronic medical record system. As part of their research agreement with Drexel University, Newport Institute provides Drexel University with de-identified data for analysis and publication; approval for use of this data for the current study was given by the treatment center, and the Drexel University IRB deemed that this was not research activity that needed IRB approval ("Not Human Subjects Research").

\section{Measures}

All variables were drawn from the Behavioral Health Screen (BHS), a tool developed by Diamond et al. (2010) to increase detection of behavioral health problems in medical settings. Questions were derived from the Diagnostic and Statistical Manual of Mental Disorders, Fourth Edition, Text Revision (DSM-IV-TR; American Psychiatric Association, 1994) criteria and other public domain psychosocial assessment tools. The BHS includes 13 modules assessing demographics, medical, school, family, safety, substance use, sexuality, depressive symptoms, anxiety, nutrition/eating, suicide, psychosis, and traumatic distress. There are 55 core questions with an additional 38 follow-up items (asked if certain core items are endorsed.) The BHS is currently used in 40 medical sites and 500 schools across Pennsylvania and is being rolled out in four other states. Psychometric validation has supported the validity and reliability of the scales (Bevans et al., 2012; Diamond et al., 2010).

\section{Parental monitoring}

Participants self-reported how often their parents knew their location on a three-point Likert-type scale ("never," "sometimes," and "often").

\section{Family conflict}

Participants self-reported frequency of arguing in the home on a three-point Likert-type scale ("never," "sometimes," and "often").

\section{Family support}

Participants self-reported frequency of turning to parents or other adult family members for support on a three-point Likert-type scale ("never," "sometimes," and "often").

\section{Parental criticism}

Participants self-reported perceived frequency of parental criticism on a five-point Likert-type scale ranging from 1 ("not critical at all") to 5 ("very critical"). 


\section{Interactions with friends}

Participants self-reported frequency of interactions with friends on a three-point Likert-type scale ("never," "sometimes," and "often").

\section{Verbal bullying}

Participants self-reported frequency of being victimized by verbal bullying on a three-point Likert-type scale ("never," "sometimes," and "often").

\section{Physical bullying}

Participants self-reported frequency of being victimized by physical bullying on a three-point Likert-type scale ("never," "sometimes," and "often").

\section{Cyberbullying}

Participants self-reported frequency of being victimized by cyberbullying on a three-point Likert-type scale ("never," "sometimes," and "often").

\section{Depressive symptoms}

Depressive symptoms were assessed using the BHS depressive symptoms subscale. This measure has shown strong reliability, factor validity, and criterion validity in previous studies (Bevans et al., 2012; Ruan-Iu et al., 2021). Using a three-point Likert-type scale ("never," "sometimes," and "often"), patients rated how often the following five depressive symptoms occurred within the past 2 weeks: consistent feelings of being down, loss of interest in things previously enjoyed, unexplained irritability or anger, loneliness, and feelings of failure. Reliability was good (alpha $=0.83$ ) in this study. Items were averaged to produce a single score for variable selection. In structural equation models, items were treated as indicators of a latent construct.

\section{Suicide risk}

Suicide risk was assessed using the BHS current suicide risk subscale. This measure has shown strong reliability, factor validity, and criterion validity in previous studies (Bevans et al., 2012). Patients rated whether they experienced suicidal ideation, made plans to commit suicide, or attempted suicide over the past two weeks (all dichotomous indicators). Reliability was acceptable (alpha $=0.79)$ in this study. For variable selection, a single dichotomous score was created indicating the presence or absence of any suicidal indicators. In structural equation models, items were treated as indicators of a latent construct.

\section{Results}

\section{Preliminary Analyses}

Mahalanobis Distance test detected and removed 13 multivariate outliers (based on combinations of age and symptom scores on depression, anxiety, substance use, and other scale scores). Means, standard deviations, and intercorrelations are found in Table 1. Approximately $72.3 \%$ of the sample reported current suicide ideation, $47.3 \%$ suicide plans, and $42.7 \%$ suicide attempts. The average depressive symptom score of 2.14 was just below the previouslyidentified cutoff of 2.20 for "moderate" depressive symptoms (Ruan-Iu et al., 2021); approximately 52.4\% of the sample was above this cutoff, and $30.8 \%$ of the sample was

Table 1 Means, standard deviations, and intercorrelations

\begin{tabular}{|c|c|c|c|c|c|c|c|c|c|c|c|}
\hline \multirow[t]{2}{*}{ Variable } & \multicolumn{10}{|c|}{ Correlations } & \multirow[t]{2}{*}{ Mean (SD) } \\
\hline & 1 & 2 & 3 & 4 & 5 & 6 & 7 & 8 & 9 & 10 & \\
\hline 1. Monitoring & & $-0.10 * *$ & $-0.23 * * *$ & -0.08 & -0.04 & $0.08 *$ & -0.05 & 0.01 & -0.01 & 0.03 & 3.27 (1.09) \\
\hline 2. Family conflict & & & $-0.12 * * *$ & $0.41 * * *$ & $0.07 *$ & $0.13 * * *$ & $0.11 * *$ & $0.12 * * *$ &, $19 * * *$ & 0.05 & $2.71(1.21)$ \\
\hline 3. Family support & & & & $-0.19 * * *$ & -0.04 & 0.00 & -0.04 & -0.03 & -0.06 & -0.04 & $1.67(1.36)$ \\
\hline 4. Parental criticism & & & & & 0.02 & $0.13 *$ & $0.11 *$ & 0.09 & $0.17 * * *$ & $0.15^{* *}$ & $2.76(1.28)$ \\
\hline 5. Interactions $\mathrm{w}$. friends & & & & & & $-0.25 * * *$ & $-0.09 * *$ & $-0.16^{* * *}$ & $-0.23 * * *$ & $-0.16 * * *$ & $2.80(1.39)$ \\
\hline 6. Verbal bullying & & & & & & & $0.42 * * *$ & $0.46 * * *$ & $0.31 * * *$ & $0.24 * * *$ & $1.30(1.45)$ \\
\hline 7. Physical bullying & & & & & & & & $0.44 * * *$ & $0.15^{* * *}$ & $0.08 *$ & $0.41(0.93)$ \\
\hline 8. Cyberbullying & & & & & & & & & $0.25 * * *$ & $0.15^{* * *}$ & $0.60(1.14)$ \\
\hline 9. Depressive symptoms & & & & & & & & & & $0.53 * * *$ & $2.14(1.25)$ \\
\hline 10. Suicide risk & & & & & & & & & & & $0.57(0.98)$ \\
\hline
\end{tabular}

Spearman's correlation coefficients are reported. All variables are scaled on a $0-4$ range except parental criticism, which ranges from 1 to 5 $* p<0.05 * * p<0.01 * * * p<0.001$ 
above the cutoff for "severe" depressive symptoms. Less than $1.3 \%$ of participants had missing data on any of the key variables; these participants were included in pairwise analyses wherever possible.

\section{Identifying Possible Main, Interactive, and Non- Linear Effects}

Sparse interaction models were estimated using hierarchical lasso in the $\mathrm{R}$ package hierNet (Tibshirani, 2020). The package hierNet tests all possible two-way interactions and quadratic effects and allows for "weak" or "strong" hierarchy to ensure that only meaningful second-order terms are included (Bien et al., 2013); in strong hierarchy, interaction terms are included in the lasso only if both constituent main effects are selected for the model, whereas in weak hierarchy, interaction terms are allowed if at least one of the main effects is selected. Weak hierarchy was specified, and 10 -fold cross-validation was used to select the best value of $\lambda$ (the regularization parameter, which determines how stringently coefficients are forced to zero) using the "lambda.1se" criterion (e.g., Soehner et al., 2019). A total of 132 possible terms were tested: 11 main effects, 110 twoway interactions, and 11 quadratic effects. Age was continuous. Gender and race were dichotomized, given the predominantly binary-gendered and white sample.

Of the predictors, all except race, parental monitoring, family support, and physical bullying were selected as main effects by the lasso procedure for depressive symptoms. Four interaction terms were selected: Family Support x Gender, Cyberbullying $x$ Gender, Interactions with Friends $\times$ Cyberbullying, and Interactions with Friends $\times$ Physical Bullying. There was also one quadratic effect, for cyberbullying; this was positive, suggesting that the impact of cyberbullying increased with frequency. As a follow-up analysis, a lasso was tested for current suicide risk; however, this only indicated gender. Therefore, nine main effects, one quadratic effect, and four interactions were included in all subsequent analyses.

\section{Interactions}

The two gender interactions are plotted (without control variables) in Fig. 1 using the $\mathrm{R}$ package "sjPlot" (Lüdecke et al., 2021). The Family Support $\times$ Gender interaction suggests that, in conditions of low family support, female respondents reported more depressive symptoms than did male respondents. At moderate or high family support, there was no difference in depressive symptoms between male and female respondents. There is a similar finding for cyberbullying; among those never cyberbullied, female respondents have increased depressive symptoms, but there is no difference among those "sometimes" or "often" cyberbullied. The two interactions between bullying and friendship are plotted (without control variables) in Fig. 2. Both suggest that youth "often" spending time with friends are at lower risk for depressive symptoms only if they are never physically or cyberbullied.

\section{Associations with Latent Depressive Symptoms and Suicide}

Then, a structural equation model was tested in the R package lavaan (Rosseel, 2012) using the diagonally weighted least squares estimator, wherein latent depressive symptoms were predicted by identified predictors, and latent suicide by the same pool plus depressive symptoms. Good fit was predetermined as $\mathrm{CFI} \geq 0.95$, SRMR $\leq 0.08$, RMSEA $\leq 0.06$ ( Hu $\&$ Bentler, 1999), and scale items were expected to have "good" loadings (above 0.55; Comrey \& Lee, 1992). Indirect effects on suicide through depressive symptoms were also tested; standard errors were computed using 5,000 bootstrap draws. All candidate variables selected were then entered into a single structural equation model, wherein all candidate predicted both depressive symptoms and suicide risk.

Overall fit was good, $\chi^{2}(103)=124.69, p=0.072 ; \mathrm{CFI}$ $=0.997$, RMSEA $=0.015$, SRMR $=0.058$. All items had "good" loadings on their factors; the lowest loading was 0.67 . The model explained $29 \%$ of the variance in depressive symptoms, and $50 \%$ of the variance in suicide. Standardized estimates are shown in Table 2. Nearly all included predictors were significant, except for cyberbullying (and its associated quadratic effect) and the Friendship $\times$ Cyberbullying interaction. Taken together, older age, female gender, family conflict, parental criticism, a lack of interactions with friends, and the experience of verbal bullying all explained unique variance in depressive symptoms. Moreover, as depicted in Figs. 1 and 2, the relationship between depressive symptoms and gender was moderated by family support and cyberbullying, and the relationship between depressive symptoms and lack of friendship interactions by physical bullying. On the other hand, outside of the sizable association between depressive symptoms and current suicide risk, only age and family conflict shared unique (and notably, negative) associations with suicide risk.

\section{Mediation by Depressive Symptoms}

Indirect effects were also tested, as shown in Table 2, indicating whether associations of family and peer factors with suicide risk were mediated by depressive symptoms. Several variables (including family conflict, parental criticism, interactions with friends, verbal bullying, age, and gender) had significant indirect effects on suicide through depressive symptoms, suggesting possible downstream associations. The final model, dropping nonsignificant 
Fig. 1 Interactions of family and peer factors with gender
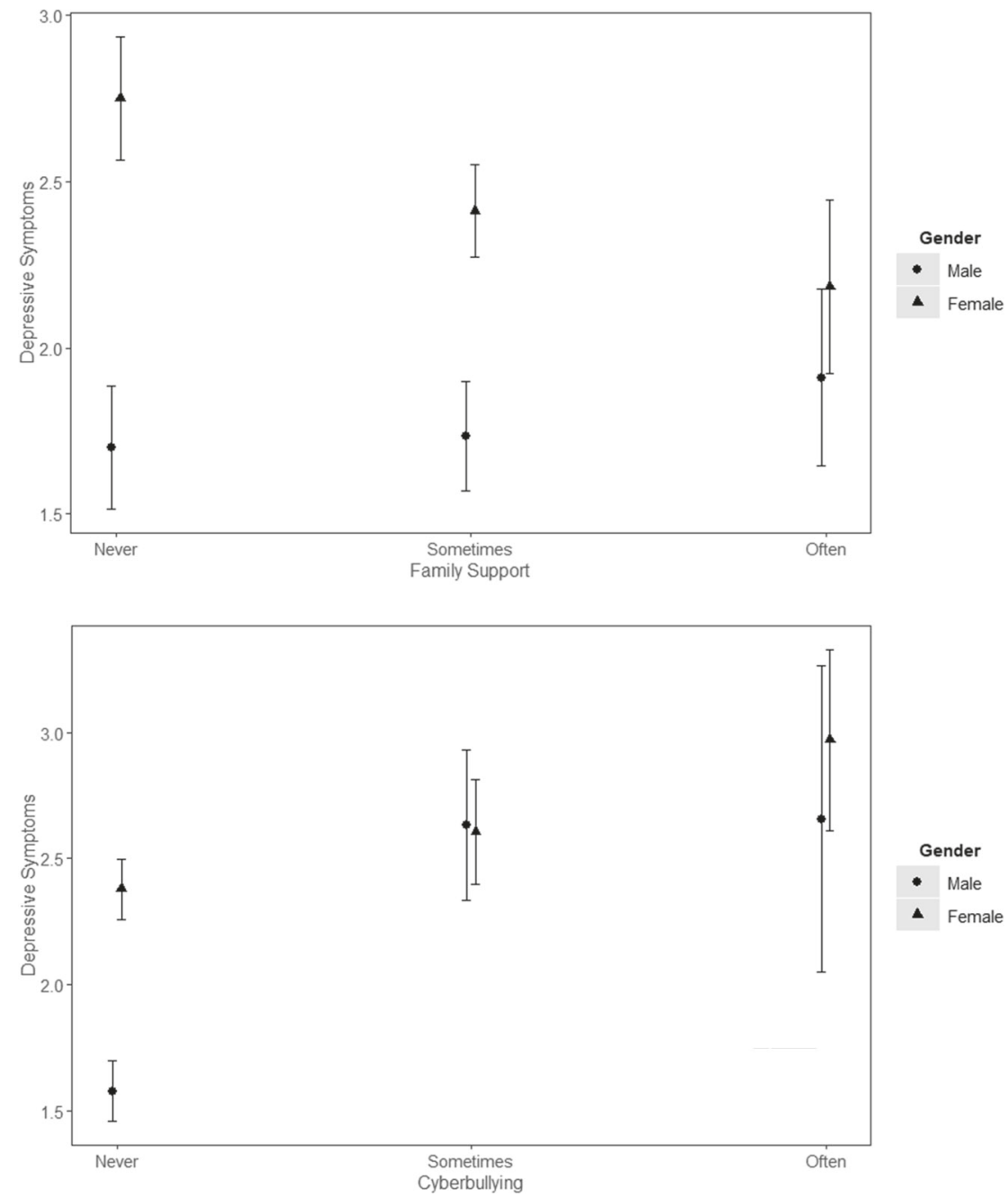

paths, is illustrated in Fig. 3. This model also fit well, $\chi^{2}$ $(108)=123.59, p=0.054 ; \mathrm{CFI}=0.996$, $\mathrm{RMSEA}=0.016$, $\mathrm{SRMR}=0.057$.

\section{Alternate Model Analyses}

Additional analyses examined the lasso model for each suicide risk indicator separately (ideation, plans, and attempts), with and without the inclusion of depressive symptoms. This did not select additional variables not already included by the depressive symptoms lasso.

\section{Discussion}

Approaches for understanding and predicting risks for adolescent depressive symptoms and suicide are still evolving. The current study used a multidimensional approach by studying the interconnected nature of family and peer influences on individual health (King et al., 2014). Following variable selection, parental criticism, family conflict, verbal bullying, and interactions with friends, alongside demographic factors of gender and age, were all found to be uniquely associated with depressive symptoms. Gender and frequency of interactions with friends were significantly moderated by other family and peer factors (family support and cyberbullying, and physical bullying, respectively). Although only family conflict and age directly predicted suicide risk above and beyond depressive symptoms, indirect associations through depressive symptoms were supported for other variables and should be explored further in longitudinal research.

There are several strengths of the current study. First, the study examined a high-risk clinical sample of adolescents and young adults, many of whom reported severe depressive symptoms and suicide risk. Therefore, family and peer factors that emerged as particularly salient in this sample are 
Fig. 2 Interactions of frequency of interactions with friends and bullying
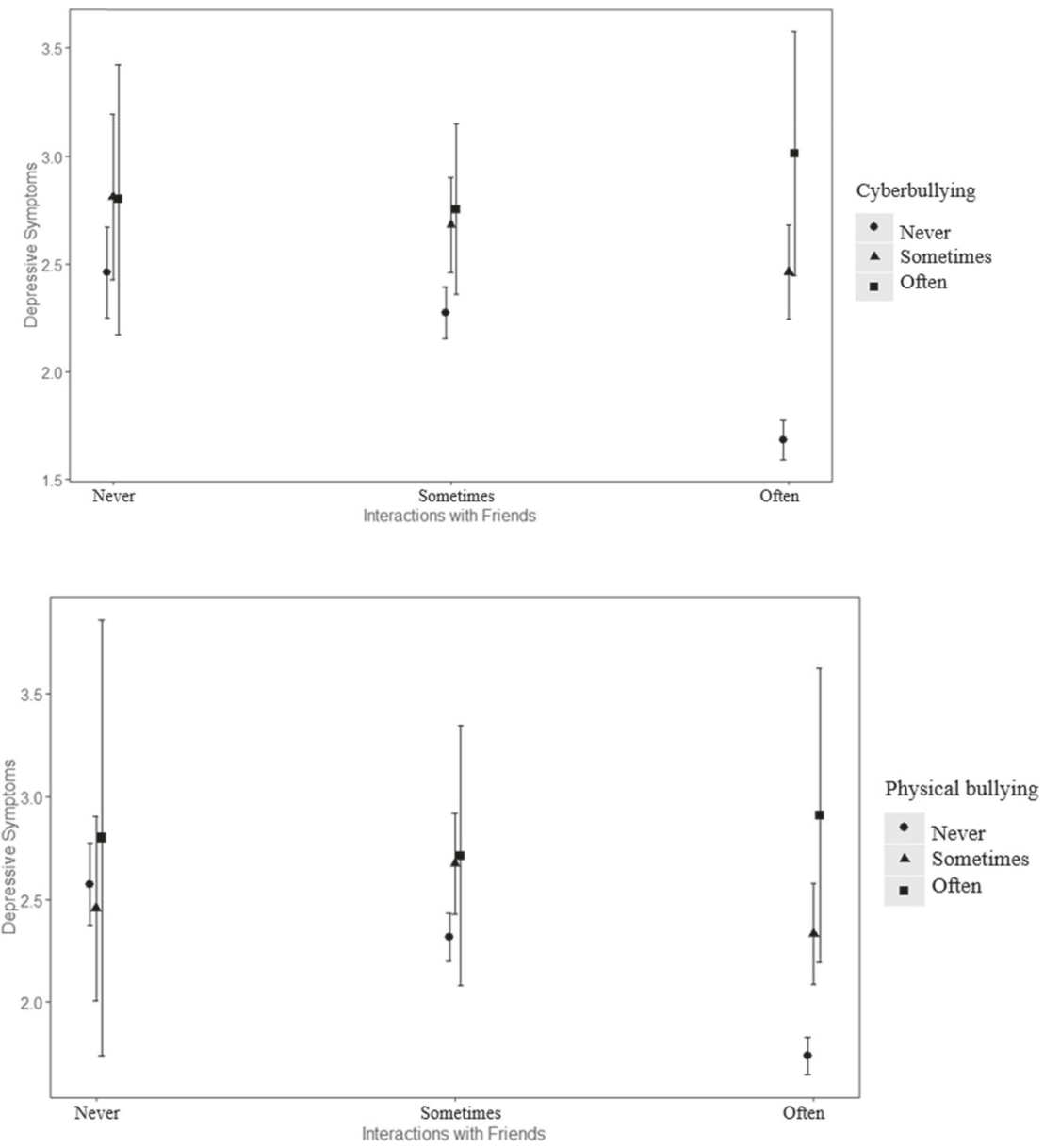

likely to be relevant for identifying those adolescents and young adults at greatest risk for severe outcomes. Although this is a cross-sectional study, better understanding of these factors may lead to advances in prevention, intervention, and treatment. Particularly in the era of COVID-19, which has greatly disrupted interpersonal relationships (Orben et al., 2020), the robust association of interactions with friends with depressive symptoms suggests that methods for developing and maintaining these potentially protective relationships are crucial for the psychological health of adolescents and young adults. Finally, the methodology of the current study also follows recent recommendations involving the use of data-driven approaches to examine multiple variables and complex relationships (Franklin et al., 2017). Given the difficulty in predicting suicide and other severe consequences of depressive symptoms, studies that examine multiple interactive risk factors are crucial for advancing understanding of how these relational processes may influence psychological well-being (Hawton et al., 2013; Restifo \& Bögels, 2009).

However, several limitations of this study should also be noted. First, the sample was highly racially homogeneous.
Although race was not selected by the lasso, this may be attributed to low power and the reduced sensitivity of this dichotomous variable. Second, only patient report with single items was used; multi-informant methods could also be used to gain a better understanding of relational processes beyond the patient's own report. Third, this study utilized a cross-sectional approach, and conclusions about directions of effects cannot be supported. Previous longitudinal research suggests that family and peer risk factors often predate mental health symptoms (Cummings et al., 2015; Jacobson \& Newman, 2016), but these associations are also likely to be bidirectional. Similarly, indirect effects suggested potential mediating pathways of family and peer factors on suicide risk through depressive symptoms, but these should not be interpreted causally. Finally, given the complex, multifactorial causes of suicide (Franklin et al., 2017), it is crucial for future research examining more proximal family and peer factors to include other categories of risk factors, including genetic factors (Levey et al., 2019) and family context (Denney, 2010), which may interact with the microsystemic social environment and depressive symptoms. 
Table 2 Standardized path estimates

\begin{tabular}{|c|c|c|c|c|}
\hline \multirow[t]{2}{*}{ Predictor } & \multirow{2}{*}{$\begin{array}{l}\text { Depressive } \\
\text { symptoms } \\
\text { Direct effects }\end{array}$} & \multicolumn{3}{|l|}{ Suicide risk } \\
\hline & & Direct effects & Indirect effects & Total effects \\
\hline Depressive symptoms & & $0.69 * * *$ & & \\
\hline \multicolumn{5}{|l|}{ Demographics } \\
\hline Age & $0.10 * *$ & $-0.10 *$ & $0.07 * *$ & -0.03 \\
\hline Gender & $0.44 * * *$ & $-\mathbf{0 . 0 3}$ & $0.30 * * *$ & $0.27 * * *$ \\
\hline \multicolumn{5}{|l|}{ Family variables } \\
\hline Family support & 0.07 & -0.06 & 0.05 & -0.01 \\
\hline Family conflict & $0.11 * *$ & $-0.11^{* *}$ & $0.07 * *$ & -0.04 \\
\hline Parental criticism & $0.12 * *$ & 0.05 & $0.08 * *$ & $0.13 * *$ \\
\hline \multicolumn{5}{|l|}{ Peer variables } \\
\hline Interactions w. friends & $-0.21 * * *$ & 0.01 & $-0.14 * * *$ & $-0.13 * *$ \\
\hline Verbal bullying & $0.22 * * *$ & 0.04 & $0.15^{* * *}$ & $0.19 * * *$ \\
\hline Physical bullying & -0.12 & 0.07 & -0.08 & 0.01 \\
\hline Cyberbullying & 0.13 & 0.02 & 0.09 & 0.11 \\
\hline Cyberbullying (quadratic) & 0.01 & -0.02 & 0.01 & -0.01 \\
\hline \multicolumn{5}{|l|}{ Interactions } \\
\hline Family Support $\times$ Gender & $-0.21 * * *$ & 0.08 & $-0.14 * *$ & -0.06 \\
\hline $\begin{array}{l}\text { Interactions w. Friends } \times \\
\text { Cyberbullying }\end{array}$ & 0.12 & -0.07 & 0.08 & -0.01 \\
\hline $\begin{array}{l}\text { Interactions w. Friends } \times \text { Phys. } \\
\text { Bullying }\end{array}$ & $0.14 *$ & -0.08 & 0.10 & -0.02 \\
\hline Gender $\times$ Cyberbullying & $-0.22 * * *$ & 0.03 & $-0.15^{* *}$ & -0.12 \\
\hline
\end{tabular}

Bolded estimates reflect terms included based on main effects in lasso model. Indirect effects are mediated by depressive symptoms. Total effects represent the sum of direct and indirect effects on suicide risk $* p<0.05 ; * * p<0.01 ; * * * p<0.001$

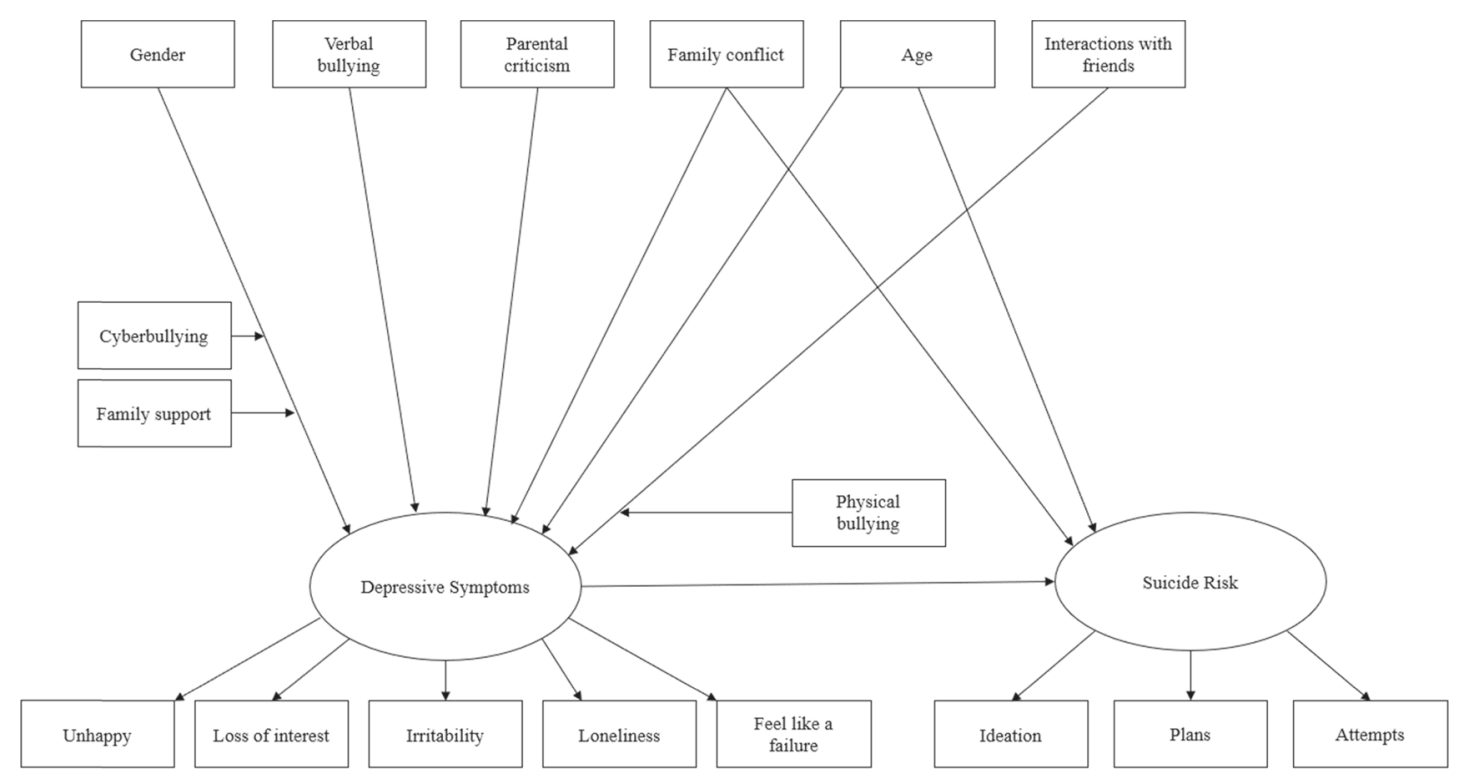

Fig. 3 Final Model. Error terms omitted for readability 
The current findings have implications for understanding how family factors related to depressive symptoms in adolescence and young adulthood. First, negative family experiences, including parental criticism and family conflict, emerged as particularly relevant for depressive symptoms. This echoes previous research (Rapp et al., 2021), but further suggests that these effects are unique; that is, independent of factors like family support, these two types of negative family experience appear to pose distinct risks. On the other hand, although the association between conflict and depressive symptoms was in the expected direction, family conflict appeared to share a negative association with suicide risk after accounting for depressive symptoms (i.e., higher conflict was associated with lower risk), suggesting more complex processes worthy of further investigation (e.g., family detachment).

Second, positive family factors (parental monitoring and family support) were not robustly associated with depressive symptoms or suicide. This may be due to the clinical severity of the sample, the developmental stage, or the specific indicators. Other studies have found mixed results regarding parental monitoring (Yap et al., 2014), and it is possible that other assessments of parental involvement may be more appropriate for older adolescents or young adults. Family support was measured by inquiring about interactional frequency (i.e., how often youth spoke with adult family members about their concerns). While measures of interactional frequency might indicate support in normative samples, families of distressed youth may be more likely to fail to respond to support-seeking or to respond negatively (Gambin et al., 2015; Preyde et al., 2011). Therefore, it is important to examine multiple dimensions of family support and cohesion in order to understand how these function among at-risk youth.

There are also important implications regarding how peer relationships are associated with depressive symptoms. In this study, interactions with friends and verbal bullying emerged as particularly salient processes for depressive symptoms. The role of peers becomes increasingly more important in adolescence and young adulthood (Magson et al., 2021; Neale et al., 2018), and feelings of acceptance or isolation from peers can be highly consequential for youth mental health. The moderation of interactions with friends by bullying (or vice versa) suggests youth with both frequent interactions with friends, and the absence of bullying, are especially unlikely to endorse depressive symptoms. On the other hand, the benefits of friendships were not moderated by family factors, in contrast to previous research (Barrera \& Garrison-Jones, 1992). However, these interactive effects have been less robust in more severe, clinical samples, perhaps due to the greater likelihood and severity of family dysfunction in these populations (Kerr et al., 2006). In the presence of these dysfunctional families, peers may serve a particularly important role in providing support and stability to distressed youth. Finally, only verbal bullying was directly relevant for depressive symptoms; this is somewhat surprising given previous research suggesting that cyberbullying poses a particularly large risk for depression (Wang et al., 2011).

\section{Conclusion}

Family and peer factors are known to be associated with youth depressive symptoms and suicide risk, but most studies examine these factors in relative isolation and without accounting for their interdependence. Without acknowledging the context in which family and peer relationship factors emerge, it is difficult to estimate the unique contributions of factors like support and conflict, particularly when the effects are not linear or depend on the levels of another factor. The current study analyzed unique, interactive, and non-linear effects of several peer and family factors associated with depressive symptoms and suicide risk in a high-risk residential sample of adolescents and young adults. Building on previous research, the current results suggest that negative family processes (like conflict and criticism) and verbal bullying, are associated with more severe, and interactions with friends less severe, depressive symptoms. Moreover, gender differences were mediated by family support and cyberbullying, and interactions with friends by physical bullying, suggesting that examining individual peer and family factors in isolation may produce misleading results. Contrary to expectations, however, few factors were directly associated with suicide risk, but several shared possible indirect pathways through depressive symptoms. These results underscore the difficulty in identifying youth with suicide risk, but also provide directions for advances in identification, research, and treatment. For high-risk adolescents and young adults, negative aspects of the family environment may be likely to outweigh any positives as distressed youth may receive support primarily from their peers. However, the increased importance of peer relationships also has a dark side; youth with a history of peer victimization may be at high risk of depressive symptoms even when they have frequent interactions with their friends. In sum, relational factors with implications for depression and suicide do not occur in a vacuum, and it is important to understand this complex microsystem to estimate the true impact of these factors on the psychological well-being of adolescents and young adults.

Authors' Contributions A.S.R. conceived of the study, performed the statistical analysis, and drafted the manuscript; J.R. helped conceive in the design and interpretation of the data; P.W. helped draft the manuscript; L.R. designed measurement and collected data and helped draft the manuscript; G.D. helped conceive in the design, provided 
supervision and resources, and helped draft the manuscript. All authors read and approved the final manuscript.

Funding Funding was provided by the Newport Institute, which has been using the Behavioral Health Screen to evaluate patient outcomes across its entire organization.

Data Sharing and Declaration This manuscript's data will not be deposited.

\section{Compliance with Ethical Standards}

Conflict of Interest The Behavioral Health Screening tool was developed by GD and colleagues but is owned by Children's Hospital of Philadelphia. They license the tool to Medical Decision Logic, Inc., a health science informatics and computer science engineering company. GD may receive a small royalty payment for his part in developing the tool. ASR and the other coauthors do not report financial interests or potential conflicts of interest.

Ethical Approval The Drexel University IRB deemed this research not requiring IRB approval ("Not Human Subjects Research").

Informed Consent Patient consent for treatment and data collection was obtained by Newport Institute at admission.

Publisher's note Springer Nature remains neutral with regard to jurisdictional claims in published maps and institutional affiliations.

\section{References}

Adams, R. E., \& Laursen, B. (2007). The correlates of conflict: disagreement is not necessarily detrimental. Journal of Family Psychology, 21(3), 445-458. https://doi.org/10.1037/0893-3200. 21.3.445.

American Psychiatric Association. (1994). Diagnostic and statistical manual of mental disorders (4th ed.). American Psychiatric Association.

Baetens, I., Claes, L., Hasking, P., Smits, D., Grietens, H., Onghena, P., \& Martin, G. (2015). The relationship between parental expressed emotions and non-suicidal self-injury: the mediating roles of self-criticism and depression. Journal of Child and Family Studies, 24(2), 491-498. https://doi.org/10.1007/s10826013-9861-8.

Baker, J. R., \& Hudson, J. L. (2013). Friendship quality predicts treatment outcome in children with anxiety disorders. Behaviour Research and Therapy, 51(1), 31-36. https://doi.org/10.1016/j. brat.2012.10.005.

Barrera, M., \& Garrison-Jones, C. (1992). Family and peer social support as specific correlates of adolescent depressive symptoms. Journal of Abnormal Child Psychology, 20(1), 1-16. https://doi. org/10.1007/BF00927113.

Bevans, K. B., Diamond, G., \& Levy, S. (2012). Screening for adolescents' internalizing symptoms in primary care: Item response theory analysis of the behavior health screen depression, anxiety, and suicidal risk scales. Journal of Developmental and Behavioral Pediatrics: JDBP, 33(4), 283-290. https://doi.org/10.1097/ DBP.0b013e31824eaa9a.

Bien, J., Taylor, J., \& Tibshirani, R. (2013). A lasso for hierarchical interactions. The Annals of Statistics, 41(3). https://doi.org/10. 1214/13-AOS1096
Bögels, S. M., \& Brechman-Toussaint, M. L. (2006). Family issues in child anxiety: attachment, family functioning, parental rearing and beliefs. Clinical Psychology Review, 26(7), 834-856. https:// doi.org/10.1016/j.cpr.2005.08.001.

Bradley, R. H., \& Corwyn, R. F. (2000). Moderating effect of perceived amount of family conflict on the relation between home environmental processes and the well-being of adolescents. Journal of Family Psychology, 14(3), 349-364. https://doi.org/ 10.1037/0893-3200.14.3.349.

Bronfenbrenner, U. (1977). Toward an experimental ecology of human development. American Psychologist, 32(7), 513-531. https:// doi.org/10.1037/0003-066X.32.7.513.

Brunstein Klomek, A., Marrocco, F., Kleinman, M., Schonfeld, I. S., \& Gould, M. S. (2007). Bullying, depression, and suicidality in adolescents. Journal of the American Academy of Child \& Adolescent Psychiatry, 46(1), 40-49. https://doi.org/10.1097/01.chi. 0000242237.84925.18.

Campos, R. C., Besser, A., \& Blatt, S. J. (2013). Recollections of parental rejection, self-criticism and depression in suicidality. Archives of Suicide Research, 17(1), 58-74. https://doi.org/10. 1080/13811118.2013.748416.

Ciairano, S., Rabaglietti, E., Roggero, A., Bonino, S., \& Beyers, W. (2007). Patterns of adolescent friendships, psychological adjustment and antisocial behavior: the moderating role of family stress and friendship reciprocity. International Journal of Behavioral Development, 31(6), 539-548. https://doi.org/10.1177/ 0165025407080573.

Compas, B. E., Jaser, S. S., Bettis, A. H., Watson, K. H., Gruhn, M., Dunbar, J. P., Williams, E., \& Thigpen, J. C. (2017). Coping, emotion regulation and psychopathology in childhood and adolescence: a meta-analysis and narrative review. Psychological Bulletin, 143(9), 939-991. https://doi.org/10.1037/bul0000110.

Comrey, A. L., \& Lee, H. B. (1992). A first course in factor analysis (2nd ed.). Psychology Press.

Cummings, E. M., Koss, K. J., \& Davies, P. T. (2015). Prospective relations between family conflict and adolescent maladjustment: security in the family system as a mediating process. Journal of Abnormal Child Psychology, 43(3), 503-515. https://doi.org/10. 1007/s10802-014-9926-1.

Denney, J. T. (2010). Family and household formations and suicide in the United States. Journal of Marriage and Family, 72(1), 202-213. https://doi.org/10.1111/j.1741-3737.2009.00692.x.

Diamond, G., Kodish, T., Ewing, E. S. K., Hunt, Q. A., \& Russon, J. M. (2021). Family processes: risk, protective and treatment factors for youth at risk for suicide. Aggression and Violent Behavior, 101586. https://doi.org/10.1016/j.avb.2021.101586

Diamond, G., Levy, S., Bevans, K. B., Fein, J. A., Wintersteen, M. B., Tien, A., \& Creed, T. (2010). Development, validation, and utility of internet-based, behavioral health screen for adolescents. Pediatrics, 126(1), e163-e170. https://doi.org/10.1542/peds. 2009-3272.

Drake, K. L., \& Ginsburg, G. S. (2012). Family factors in the development, treatment, and prevention of childhood anxiety disorders. Clinical Child and Family Psychology Review, 15(2), 144-162. https://doi.org/10.1007/s10567-011-0109-0.

Franklin, J. C., Ribeiro, J. D., Fox, K. R., Bentley, K. H., Kleiman, E. M., Huang, X., Musacchio, K. M., Jaroszewski, A. C., Chang, B. P., \& Nock, M. K. (2017). Risk factors for suicidal thoughts and behaviors: a meta-analysis of 50 years of research. Psychological Bulletin, 143(2), 187-232. https://doi.org/10.1037/bul0000084.

Gambin, M., Gambin, T., \& Sharp, C. (2015). Social cognition, psychopathological symptoms, and family functioning in a sample of inpatient adolescents using variable-centered and person-centered approaches. Journal of Adolescence, 45, 31-43. https://doi.org/ 10.1016/j.adolescence.2015.08.010. 
Gould, M. S., \& Kramer, R. A. (2001). Youth suicide prevention. Suicide \& Life-Threatening Behavior, 31(Supplement), 37.

Hawton, K., Casañas i Comabella, C., Haw, C., \& Saunders, K. (2013). Risk factors for suicide in individuals with depression: a systematic review. Journal of Affective Disorders, 147(1), 17-28. https://doi.org/10.1016/j.jad.2013.01.004.

Hu, L., \& Bentler, P. M. (1999). Cutoff criteria for fit indexes in covariance structure analysis: conventional criteria versus new alternatives. Structural Equation Modeling: A Multidisciplinary Journal, 6(1), 1-55. https://doi.org/10.1080/10705519909540118.

Jacobson, N. C., \& Newman, M. G. (2016). Perceptions of close and group relationships mediate the relationship between anxiety and depression over a decade later. Depression and Anxiety, 33(1), 66-74. https://doi.org/10.1002/da.22402.

Kaltiala-Heino, R., Fröjd, S., \& Marttunen, M. (2009). Involvement in bullying and depression in a 2-year follow-up in middle adolescence. European Child \& Adolescent Psychiatry, 19(1), 45 https://doi.org/10.1007/s00787-009-0039-2.

Kerr, D. C. R., Preuss, L. J., \& King, C. A. (2006). Suicidal adolescents' social support from family and peers: gender-specific associations with psychopathology. Journal of Abnormal Child Psychology, 34(1), 99-110. https://doi.org/10.1007/s10802005-9005-8.

King, M. F., Renó, V. F., \& Novo, E. M. L. M. (2014). The concept, dimensions and methods of assessment of human well-being within a socioecological context: a literature review. Social Indicators Research, 116(3), 681-698. https://doi.org/10.1007/ s11205-013-0320-0.

Kurdek, L. A., \& Fine, M. A. (1994). Family acceptance and family control as predictors of adjustment in young adolescents: linear, curvilinear, or interactive effects? Child Development, 65 (4), 1137-1146. https://doi.org/10.1111/j.1467-8624.1994. tb00808.x.

Levey, D. F., Polimanti, R., Cheng, Z., Zhou, H., Nuñez, Y. Z., Jain, S., He, F., Sun, X., Ursano, R. J., Kessler, R. C., Smoller, J. W., Stein, M. B., Kranzler, H. R., \& Gelernter, J. (2019). Genetic associations with suicide attempt severity and genetic overlap with major depression. Translational Psychiatry, 9(1), 1-12. https://doi.org/10.1038/s41398-018-0340-2.

Lewis, A. J., Kremer, P., Douglas, K., Toumborou, J. W., Hameed, M. A., Patton, G. C., \& Williams, J. (2015). Gender differences in adolescent depression: differential female susceptibility to stressors affecting family functioning. Australian Journal of Psychology, 67(3), 131-139. https://doi.org/10.1111/ajpy.12086.

Lüdecke, D., Bartel, A., Schwemmer, C., Powell, C., Djalovski, A., \& Titz, J. (2021). sjPlot: data visualization for statistics in social science (2.8.9) [Computer software]. https://CRAN.R-project.org/ package $=$ sjPlot

Magson, N. R., Freeman, J. Y. A., Rapee, R. M., Richardson, C. E., Oar, E. L., \& Fardouly, J. (2021). Risk and protective factors for prospective changes in adolescent mental health during the COVID-19 pandemic. Journal of Youth and Adolescence, 50(1), 44-57. https://doi.org/10.1007/s10964-020-01332-9.

Mojtabai, R., Olfson, M., \& Han, B. (2016). National trends in the prevalence and treatment of depression in adolescents and young adults. Pediatrics, 138(6). https://doi.org/10.1542/peds.20161878

National Center for Injury Prevention and Control (U.S.). Division of Violence Prevention. (Ed.). (2015). Suicide: facts at a glance, 2015 (cdc:34181). https://stacks.cdc.gov/view/cdc/34181

Neale, J., Tompkins, C. N. E., \& Strang, J. (2018). Qualitative exploration of relationships between peers in residential addiction treatment. Health \& Social Care in the Community, 26(1), e39-e46. https://doi.org/10.1111/hsc.12472.

Orben, A., Tomova, L., \& Blakemore, S.-J. (2020). The effects of social deprivation on adolescent development and mental health.
The Lancet Child \& Adolescent Health, 4(8), 634-640. https:// doi.org/10.1016/S2352-4642(20)30186-3.

Parker, G., \& Brotchie, H. (2010). Gender differences in depression. International Review of Psychiatry, 22(5), 429-436. https://doi. org/10.3109/09540261.2010.492391.

Preyde, M., Cameron, G., Frensch, K., \& Adams, G. (2011). Parent-child relationships and family functioning of children and youth discharged from residential mental health treatment or a home-based alternative. Residential Treatment for Children \& Youth, 28(1), 55-74. https://doi.org/10.1080/0886571X.2011. 550171.

Prinstein, M. J., Boergers, J., Spirito, A., Little, T. D., \& Grapentine, W. L. (2000). Peer functioning, family dysfunction, and psychological symptoms in a risk factor model for adolescent inpatients' suicidal ideation severity. Journal of Clinical Child Psychology, 29(3), 392-405. https://doi.org/10.1207/ S15374424JCCP2903_10.

Queen, A. H., Stewart, L. M., Ehrenreich-May, J., \& Pincus, D. B. (2013). Mothers' and fathers' ratings of family relationship quality: associations with preadolescent and adolescent anxiety and depressive symptoms in a clinical sample. Child Psychiatry \& Human Development, 44(3), 351-360. https://doi.org/10.1007/ s10578-012-0329-7.

Randell, B. P., Wang, W.-L., Herting, J. R., \& Eggert, L. L. (2006). Family factors predicting categories of suicide risk. Journal of Child and Family Studies, 15(3), 247-262. https://doi.org/10. 1007/s10826-006-9020-6.

Rapp, A. M., Chavira, D. A., Sugar, C. A., \& Asarnow, J. R. (2021). Incorporating family factors into treatment planning for adolescent depression: perceived parental criticism predicts longitudinal symptom trajectory in the Youth Partners in Care trial. Journal of Affective Disorders, 278, 46-53. https://doi.org/10.1016/j.jad. 2020.09.028.

Restifo, K., \& Bögels, S. (2009). Family processes in the development of youth depression: translating the evidence to treatment. Clinical Psychology Review, 29(4), 294-316. https://doi.org/10.1016/ j.cpr.2009.02.005.

Rice, F., Harold, G. T., Shelton, K. H., \& Thapar, A. (2006). Family conflict interacts with genetic liability in predicting childhood and adolescent depression. Journal of the American Academy of Child \& Adolescent Psychiatry, 45(7), 841-848. https://doi.org/10. 1097/01.chi.0000219834.08602.44.

Rosseel, Y. (2012). lavaan: An R package for structural equation modeling. Journal of Statistical Software, 48(1), 1-36. https:// doi.org/10.18637/jss.v048.i02.

Ruan-Iu, L., Chod, D., Weissinger, G., VanFossen, C., Saionz, R., Tien, A., \& Diamond, G. S. (2021). Validation of the Behavioral Health Screen-Depression with adolescents in residential care. Manuscript under review at European Journal of Psychological Assessment (under review).

Schwartz-Mette, R. A., Shankman, J., Dueweke, A. R., Borowski, S., \& Rose, A. J. (2020). Relations of friendship experiences with depressive symptoms and loneliness in childhood and adolescence: a meta-analytic review. Psychological Bulletin, 146(8), 664-700. https://doi.org/10.1037/bu10000239.

Sheeber, L., Hops, H., \& Davis, B. (2001). Family processes in adolescent depression. Clinical Child and Family Psychology Review, 4 (1), 19-35. https://doi.org/10.1023/a:1009524626436.

Sheftall, A. H., Mathias, C. W., Furr, R. M., \& Dougherty, D. M. (2013). Adolescent attachment security, family functioning, and suicide attempts. Attachment \& Human Development, 15(4), 368-383. https://doi.org/10.1080/14616734.2013.782649.

Soehner, A. M., Bertocci, M. A., Levenson, J. C., Goldstein, T. R., Rooks, B., Merranko, J., Hafeman, D., Diler, R., Axelson, D., Goldstein, B. I., Hickey, M. B., Monk, K., Phillips, M. L., \& Birmaher, B. (2019). Longitudinal associations between sleep 
patterns and psychiatric symptom severity in high-risk and community comparison youth. Journal of the American Academy of Child \& Adolescent Psychiatry, 58(6), 608-617. https://doi. org/10.1016/j.jaac.2018.09.448.

Sun, R. C. F., \& Hui, E. K. P. (2007). Psychosocial factors contributing to adolescent suicidal ideation. Journal of Youth and Adolescence, 36(6), 775-786. https://doi.org/10.1007/s10964006-9139-1.

Tibshirani, J. B. and R. (2020). hierNet: a Lasso for Hierarchical Interactions (1.9) [Computer software]. https://CRAN.R-project. org $/$ package $=$ hierNet

Ueno, K. (2005). The effects of friendship networks on adolescent depressive symptoms. Social Science Research, 34(3), 484-510. https://doi.org/10.1016/j.ssresearch.2004.03.002.

Vieno, A., Santinello, M., Pastore, M., \& Perkins, D. D. (2007). Social support, sense of community in school, and self-efficacy as resources during early adolescence: an integrative model. American Journal of Community Psychology, 39(1-2), 177-190. https://doi.org/10.1007/s10464-007-9095-2.

Vitaro, F., Boivin, M., \& Bukowski, W. M. (2009). The role of friendship in child and adolescent psychosocial development. In Handbook of peer interactions, relationships, and groups (pp. 568-585). The Guilford Press.

Wang, J., Nansel, T. R., \& Iannotti, R. J. (2011). Cyber and traditional bullying: differential association with depression. Journal of Adolescent Health, 48(4), 415-417. https://doi.org/10.1016/j.ja dohealth.2010.07.012.

Yap, M. B. H., Pilkington, P. D., Ryan, S. M., \& Jorm, A. F. (2014). Parental factors associated with depression and anxiety in young people: a systematic review and meta-analysis. Journal of Affective Disorders, 156, 8-23. https://doi.org/10.1016/j.jad. 2013.11.007.

Yu, S., Clemens, R., Yang, H., Li, X., Stanton, B., Deveaux, L., Lunn, S., Cottrell, L., \& Harris, C. (2006). Youth and parental perceptions of parental monitoring and parent-adolescent communication, youth depression, and youth risk behaviors. Social Behavior and Personality: An International Journal, 34(10), 1297-1310. https://doi.org/10.2224/sbp.2006.34.10.1297.

Alannah Shelby Rivers is a post-doctoral researcher at the Center for Family Intervention Science, Drexel University. Her major research interests include the influence of close relationships on mental and physical health, and psychometrics.

Jody Russon is an Assistant Professor of Human Development and Family Science at Virginia Polytechnic Institute and State University. Her major research interests focus on vulnerable youth, particularly LGBTQ + adolescents and young adults.

Payne Winston-Lindeboom is a project coordinator at the Center for Family Intervention Science, Drexel University. Her major research interests are in the mental and behavioral health of adolescents and young adults, especially those who have dealt with family issues or trauma.

Linda Ruan-lu is a post-doctoral fellow at the Center for Family Intervention Science at Drexel University. Her major research interests focus on cross-cultural assessment, psychiatric diagnosis and assessment, and suicidal behavior among youth.

Guy Diamond is an Associate Professor in the Department of Counseling and Family Therapy in the College of Nursing and Health Professions at Drexel University and the director of the Center for Family Intervention Science. His major research interests include youth suicide prevention and treatment, including attachment-based family therapy, specially developed and tested for treating youth depression and suicide. 